\title{
Multiple-Node Models of Asynchronous Wind Turbines in Wind Farm for Load Flow Analysis
}

\author{
Jianxue Wang ${ }^{1}$, Chengchen Huang ${ }^{2}$ and Ahmed F. Zobaa ${ }^{3}$ \\ ${ }^{1}$ School of Electrical Engineering, Xi'an Jiaotong University, Xi'an, Shaanxi, \\ 710049, People's Republic of China \\ ${ }^{2}$ Jiangsu Electric Power Company Economic Research Institute, Nanjing, Jiangsu, \\ 210008, People's Republic of China \\ ${ }^{3}$ College of Engineering, Design and Physical Sciences; Brunel University London; \\ Uxbridge; UK
}

\begin{abstract}
Considering load flow analysis of power system integrated with multiple wind generators (WGs), the Multiple-Node Models of an asynchronous generator are proposed in this paper. It is more convenient to use the Multiple-Node Models for load flow problem, because the models can be directly applied to conventional load flow programs, and the iteration process of the rotor slip is no longer needed. In addition, a kind of aggregation methodology is presented to aggregate a wind farm into multiple WGs in this paper. The case study is carried out on the basis of the modified IEEE-30 test system, and the detailed comparisons are given. The results show the effective performance of the proposed methods.
\end{abstract}

Key words load flow analysis, multiple-node models, wind farm aggregation

\section{Introduction}

Wind energy is considered plentiful, renewable and clean. The utilization of wind power in electric

Address correspondence to Dr. Jianxue Wang. School of Electrical Engineering, Xi'an Jiaotong University, Xi'an, 710049, P.R. China. Email: jxwang@mail.xjtu.edu.cn 
power system is gaining popularity due to its economic and social benefits. However, wind power generation introduces many problems into power systems because of its natural fluctuation and intermittency. For example, it has perturbed the grid operation on the aspects of power security and stability and its intermittency also brought challenges to electricity markets involving wind power trading [1]. For power systems including wind farms, load flow analysis is often used to assess the impact of wind turbines on the grid's steady-state operation and lay a foundation for other theoretical studies such as grid stability [2]. In load flow analysis, all the buses are divided into three types: Slack buses, PQ buses and PV buses. Load flow calculation derives primarily the voltage magnitudes and angles of all buses. Afterwards, given branch impedances, the active and reactive power flowed through branches and power losses can be calculated.

Considering load flow analysis, models for asynchronous generator in steady-state have been proposed and discussed in many literatures. After defining the model of WGs in the system, one can then adopt iterative algorithm, i.e. Newton-Raphson algorithm, to solve load flow problems. As a summary, the existing methods for deterministic load flow analysis of a system containing WGs can be sorted as the following cases:

In general, an asynchronous WG has a short-circuited rotor and it works at the super synchronous state [3]. The PQ Model studied treats WG as a node of which the active and reactive power are known quantities [4]-[7]. It takes some approximations by adopting the assumption $Q_{e}=f\left(P_{e}\right)$, here the symbol $\boldsymbol{f}$ represents a general quadratic function[5]. In reference [8] a creative improvement is presented for the PQ Model: a more accurate simulating method of asynchronous WGs was researched in detail by using some circuit transformations.

In comparison, an exactly accurate model was also researched for asynchronous WGs in [5], [6], called RX Model, which considers the machine's internal circuit parameters by using the $\Gamma$ circuit model of a WG. With the Thevenin theorem applied, another more accurate RX Model, i.e. RX2, which is based on the T circuit model, was discussed in [9]. The conventional RX Models are often carried out through a sequential iterative approach. The authors advice that RX Models can be also 
solved in a unified way by adding a mismatch equation in the load flow formulation [10]. In [11], wind farms and automatic load-frequency control are both taken into account in power mismatch equations.

A new model is explored by considering the induction generator equivalent circuit without rotor slip. Paper [12] derives the active and reactive power outputs of wind turbine generating systems as functions of terminal voltages, mechanical power and winding parameters except the rotor slip. The proposed model is compared with existing models by mathematical computation. Paper [13] incorporates the same model into distribution system load flow analysis and compared with Matlab/Simulink models.

In this article, a Multiple-Node Models for the load flow analysis of an asynchronous WG is proposed. Compared with those models mentioned in the previous literatures [4]-[13], the proposed model can be directly performed on any conventional load flow programs without additional modifications on original codes or the iterative processes for slip $s$ and reactive power. What's more, it accelerates the program computation speed because of its brevity. For comparison, this article simplifies the RX model in [5] by assuming the active power only relates to wind speed. It turns out that the proposed model has equal accuracy as PQ model and the simplified RX model. By using this model, load flow analysis of a power system integrated with wind farms can be more efficient and practical.

The remainder of this paper is structured as below: Section 2 develops the Multiple-Node Models of an asynchronous generator for load flow calculation after a discussion of its basic model. Then a wind farm aggregation methodology is presented in detail. Section 3 shows the load flow results of the modified IEEE-30 test system by using conventional and proposed models respectively, with wind aggregation applied, and the comparisons are given. In Section 4 the conclusions are drawn. 


\section{Load flow model of asynchronous wind generator}

\subsection{Steady-state WG Models}

In terms of wind turbines with certain blades, the mechanical power $P_{m}$ is a function of the wind speed $v_{w}$. The relationship between $P_{m}$ and $v_{w}$ is shown as follows:

$$
P_{m}=\frac{1}{2} \rho A v_{w}^{3} C_{p}
$$

Where

$\rho$ is the air density $\left(\mathrm{kg} / \mathrm{m}^{3}\right)$

$A$ is the blades sweeping area $\left(\mathrm{m}^{2}\right)$

$C_{p}$ is the power coefficient.

$v_{w}$ is the wind speed $(\mathrm{m} / \mathrm{s})$.

If wind speed $v_{w}$ is given, the mechanical power $P_{m}$ generated by the wind power could be obtained as a constant known quantity in the following analysis. In general, the manufactures also supply the curve of the mechanical power. Therefore $P_{m}$ can be treated as the input data of the load flow problem.

Figure 1 shows the per-phase equivalent circuit of an asynchronous WG referred to the stator-winding.

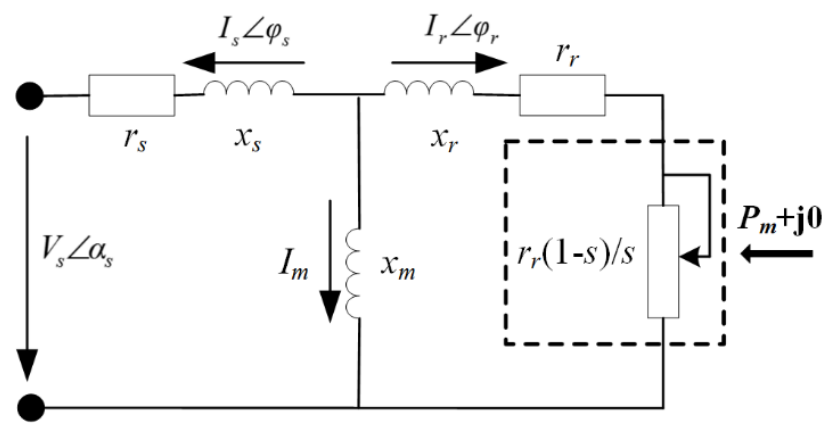

Figure 1. The equivalent electrical circuit of a WG.

Where $s$ is the slip, and $r_{r}, r_{s}$ are rotor and stator resistance. $x_{r}, x_{s}$ are rotor and stator reactance and $x_{m}$ is the magnetizing reactance. It needs to be declared that in this research the approximation 
of neglecting the core loss is adopted. If the core loss is to be considered, it only needs to add a by-pass resistance $R_{f e}$ in parallel with the magnetizing reactance $x_{m}$.

In Figure 1, the rotor is considered locked and the mechanical power on the shaft $P_{m}$ is represented by the active power consumed on the variable resistance, as expressed in Eq. (2).

$$
P_{m}=-I_{r}^{2} \cdot \frac{1-s}{s} r_{r}
$$

Inversely the active power consumed on the variable resistance in Figure 1 can be replaced by mechanical power $P_{m}$ of known rotor speed. Hence we could use an equivalent constant power source to substitute the variable resistance shown as the dashed box in Figure 1.

With such a transformation, Eq. (2) can be eliminated from the load flow equations and the solution of the slip $s$ can be decoupled from the load flow calculation.

\subsection{Multiple-Node Models of a WG}

In practice, wind speed data and wind turbine parameters are regarded as known quantities. Hence under the precondition of adopting certain control strategies for fixed speed induction generators, the mechanical power of the WG can be seen as a constant input variable.

Developing from the analysis above, the model of an asynchronous machine has been transformed into the following proposed conventional load flow model. Figure 2 shows the Three-Node Model of WG which is connected to a power system.

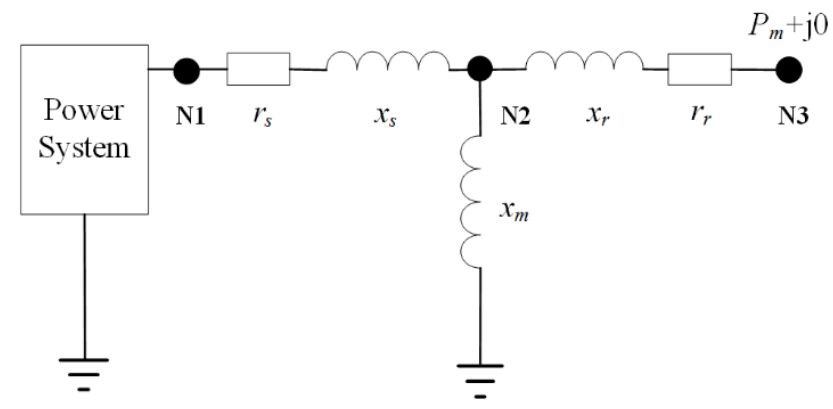

Figure 2. Three-Node Model of a WG.

Three PQ buses and several virtual branches are added to simulate a WG bus. In Figure 2, N1, N2 and $\mathbf{N 3}$ are the three equivalent buses of a WG. For load flow analysis, in the nodal admittance 
matrix, a three-order matrix is used for a WG bus, and the values of the injected power are given in Eq. (3).

$$
\begin{aligned}
& P_{3}=P_{m} \\
& P_{1}=Q_{1}=P_{2}=Q_{2}=Q_{3}=0
\end{aligned}
$$

It can be seen that $\mathbf{N} 1$ and $\mathbf{N} \mathbf{2}$ are both contact nodes, that is, they are PQ buses with $\mathrm{P}=0$ and $\mathrm{Q}=0$. Figure 2 shows a power system which connects with only one WG. If there are amounts of WGs, for instance, assuming that the number of WGs is $N$, then it needs $3 N$ PQ buses and $3 N$ branches to simulate the all WGs, with the Three-Node Model adopted.

In addition, through such a Y- $\triangle$ transformation of the circuit, the Three-Node model can be converted into the Two-Node model conveniently. Under the circumstance of very large amount of wind generators connected to the power system, using the Two-Node Model may reduce the size of power flow matrix than using the Three-Node Model. However, the Three-Node model has definite physical meaning, so this paper still investigates the Three-Node Model in the following discussion.

\subsection{Multiple-Node Models for Load Flow Analysis}

The steady-state model of a wind farm is often used to load flow calculation. With the Multiple-Node Models adopted, potential researches on power systems integrated with asynchronous WGs will be much more convenient and efficient. The next is to present the detailed implementation of the Multiple-Node Models for load flow analysis.

The modification for load flow analysis is applied easily in terms of Three-Node Model. For example, as shown in Figure 2, a WG is connected to a given power system. Given the mechanical power and certain working condition, the load flow problem containing wind power can be solved as follows:

1) to add three PQ buses with detailed parameters shown in Table 1;

2) to add two branches with the detailed parameters shown in Table 2. 


\section{TABLE 1}

The parameters of the bus data for WG bus

\begin{tabular}{lcccccc}
\hline \multirow{3}{*}{ Bus } & $\begin{array}{c}\text { Real } \\
\text { power } \\
\text { output }\end{array}$ & $\begin{array}{c}\text { Reactive } \\
\text { power } \\
\text { output }\end{array}$ & $\begin{array}{c}\text { Real } \\
\text { power } \\
\text { demand }\end{array}$ & $\begin{array}{c}\text { Reactive } \\
\text { power } \\
\text { demand }\end{array}$ & $\begin{array}{c}\text { Shunt } \\
\text { conductance }\end{array}$ & $\begin{array}{c}\text { Shunt } \\
\text { susceptance } \\
B_{g}\end{array}$ \\
$P_{g}$ & $Q_{g}$ & $P_{d}$ & $Q_{d}$ & $G_{s}$ & $B_{s}$ \\
\hline N1 & 0 & 0 & 0 & 0 & 0 & 0 \\
N2 & 0 & 0 & 0 & 0 & 0 & $-1 / x_{m}$ \\
N3 & $P_{m}$ & 0 & 0 & 0 & 0 & 0 \\
\hline
\end{tabular}

\section{TABLE 2}

The parameters of the added branch data for WG bus

\begin{tabular}{lllc}
\hline Branch & Line resistance $r$ & Line reactance $x$ & Total line charging $b$ \\
\hline From N1 to N2 & $r_{s}$ & $x_{s}$ & 0 \\
From N2 to N3 & $r_{r}$ & $x_{r}$ & 0 \\
\hline
\end{tabular}

Obviously, the virtual bus definition of WG keeps consistent with that of the general bus. The proposed models facilities the load flow analysis of a power system which contains large-scale wind farms: the calculation can be directly carried out based on the existing conventional load flow program. Compared with the general PQ model, these models take into detailed consideration the active power consumed by winding resistances and the reactive power absorbed from the grid. In the meanwhile, they also possess the advantages of simplicity and convenience for interfacing with universal power flow programs.

It can be seen that load flow based on Multiple-Node Models needs not concern about the iteration process of $s$ which spares the burden of calculation. On the contrary, in conventional RX Models [9], $s$ is a key variable for iteration and it is usually derived in a very complex expression, which may take more computation time. Anyway, if the value of $s$ needs to be known in some occasions, it can be also obtained conveniently after the load flow is solved. When Three-Node 
Model adopted, the value of the slip $s$, can be calculated by Eq. (4).

$$
s=\frac{I_{r}^{2} r_{r}}{I_{r}^{2} r_{r}-P_{m}}
$$

In Eq. (4), the value of $I_{r}$ can be got from the results of the load flow calculation by (5).

$$
I_{r}=\frac{V_{2}-V_{3}}{r_{r}+\mathrm{j} x_{r}}
$$

In Eq. (5), the value of $\boldsymbol{V}_{2}$ and $\boldsymbol{V}_{3}$ are the phasors of the voltage of $\mathbf{N} \mathbf{2}$ and $\mathbf{N} \mathbf{3}$ in Figure 2.

For convenience, another method to calculate the value of $I_{r}^{2} r_{r}$ is to use the active power loss consumed on the branch between $\mathbf{N} \mathbf{2}$ and $\mathbf{N 3}$, when the branch power flow results are printed out, the value of $s$ can be directly obtained by Eq. (4).

\subsection{Wind farm aggregation study}

In steady-state analysis, it is more important for the power grid side to concern about the global state of a wind farm in order to reduce computation time. The research of the wind farm aggregation in this section is to replace the whole wind farm by several equivalent WGs on the premise of acceptable accuracy. As shown in Figure 3, the wind farm incorporates amounts of WGs, and each of them is connected by a box-type transformer. The high-voltage terminals of the box-type transformer are gathered by the collector buses of the wind farm.

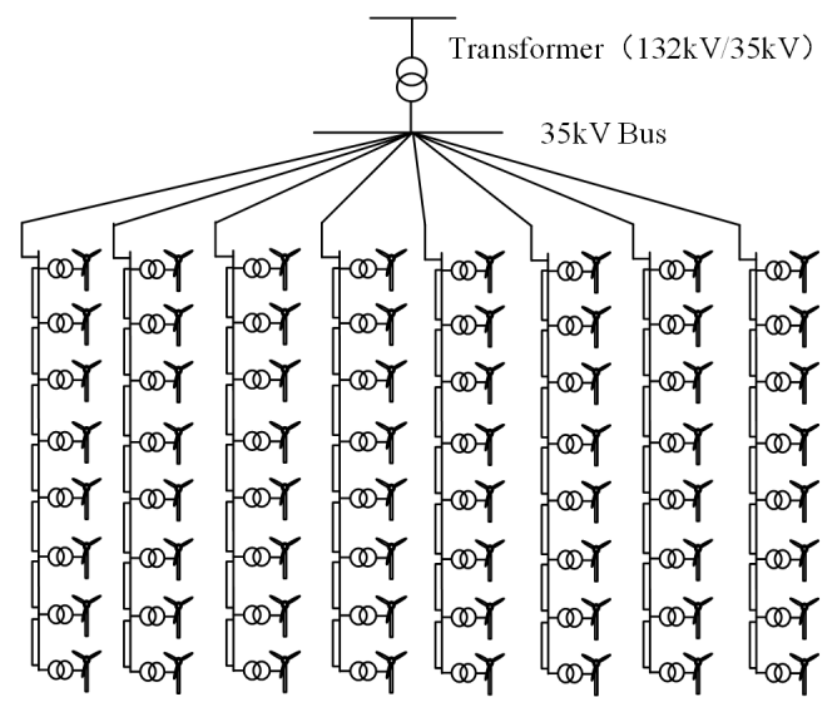

Figure 3. The wind farm diagram 
As long as the mechanical powers of the WGs in the $i^{\text {th }}$ row are determined by wind speed, their summation can be treated as the $i^{\text {th }}$ equivalent WG mechanical power, written as $P_{m, i, \Sigma}$. If the farm contains $N$ rows of WGs and there are $M$ WGs in each row, then the expression of $P_{m, i, \Sigma}$ can be shown as in Eq. (6).

$$
P_{m, i, \Sigma}=\sum_{j=1}^{M} P_{m_{-} i, j}
$$

Where $P_{m_{-} i, j}$ is the mechanical power of the $j^{\text {th }}$ WG on the $i^{\text {th }}$ row.

Wind farm aggregation is practically needed but it is not the key point of this paper. There are various aggregation methods when different aspects, i.e. wind speed, are concerned [14]. In this article, we apply a simple method based on some assumptions. Considering the working points of WGs are close, there is no voltage difference among the WGs in one row. The collector buses can be replaced by one equivalent collector bus. The aggregation criterion is that, seeing from the grid side, the virtual branch impedance consumes the same power loss as that along an actual branch [15]. Since the WGs and box-type transformers in one wind farm are usually of the same type, their electrical parameters are also consistent. So the parallel equivalent method is used to aggregate WGs and box-type transformers in all the $N$ rows of the wind farm. Considering that in the $i^{\text {th }}$ row $M$ adjacent WGs are in parallel, electrical parameters of the equivalent WG $r_{s \Sigma}, r_{r \Sigma}, x_{s \Sigma}, x_{r \Sigma}, x_{m \Sigma}$ is $1 / M$ of the parameters of the actual WG. Similarly, $R_{T}+\mathrm{j} X_{T}$, the electrical impedance of the equivalent transformer, is $1 / M$ of the parameters of the box-type transformer.

Afterwards, a wind farm with $n$ rows can be simplified into $n$ WGs connected to the wind bus as described by Figure 4. Every WG row of the wind farm contains an equivalent WG with corresponding lines. The impendence of the line between the terminal of the $i^{\text {th }}$ row and the wind farm bus is $r_{i}+\mathrm{j} x_{i}$. 


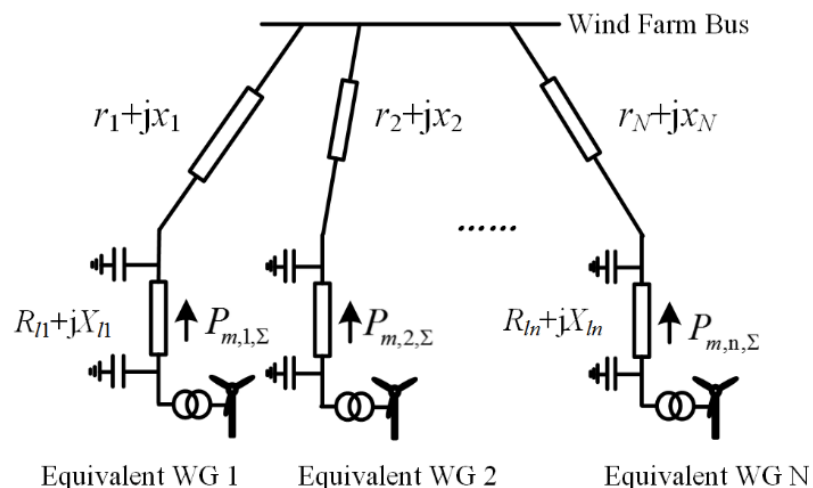

Figure 4. The equivalent model of the whole wind farm

\section{Case Study}

The modified IEEE-30 test system [16] is applied in the case study, which is shown in Figure 5.

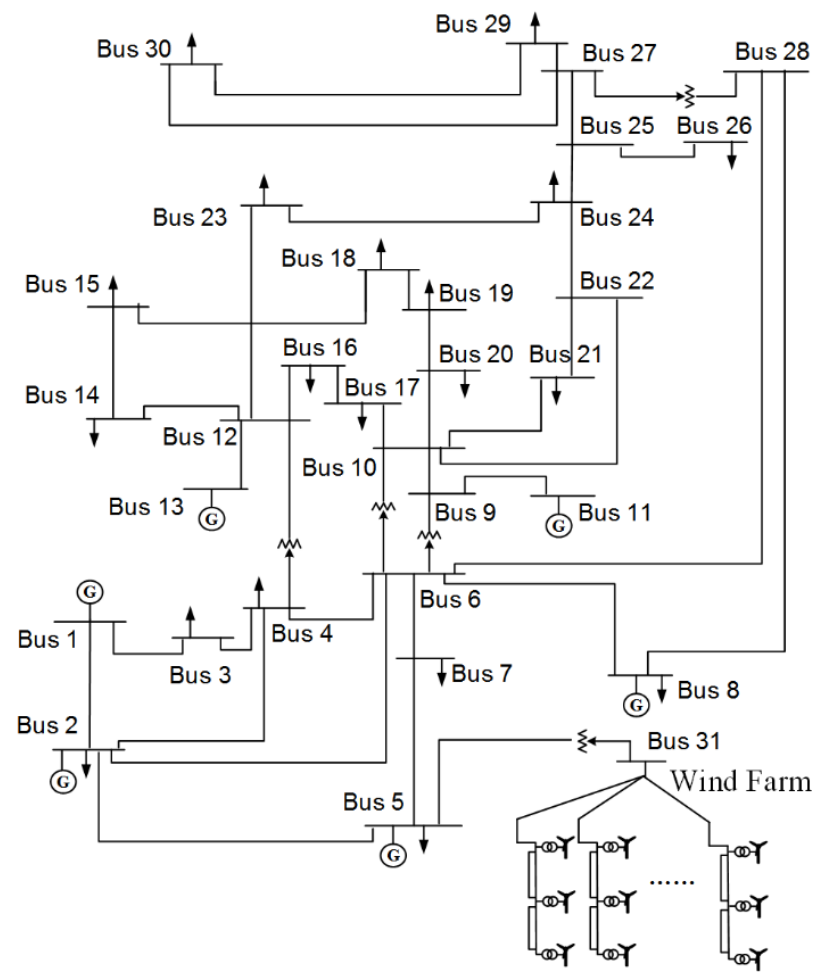

Figure 5. The diagram of the modified IEEE-30 test system.

In the IEEE-30 test system, a new added bus, Bus 31, is added to connect the wind farm. In the bottom of Figure 5, Bus 31 is connected to Bus 5 through a transformer where the impedance of the added transformer is $0.002+\mathrm{j} 0.08$ (p.u.), the ratio is 1.0 (p.u.). 
Inside the wind farm, the WGs are connected by the collector buses which are assumed to be overhead lines. The distance between the adjacent WGs in one row is $200 \mathrm{~m}$ and the distance between the adjacent rows is $300 \mathrm{~m}$. Each row is connected by a $4 \mathrm{~km}$ overhead line to the wind farm Bus 31. The impedance of the overhead line is $(0.005+\mathrm{j} 0.0126)(\mathrm{p} . \mathrm{u}.) / \mathrm{km}$, and the charging admittance is neglected.

The wind farm has 8 rows of WGs and each row is made up of 8 WGs with their box-type transformers. As for the WG, the impedance on the stator side is $0.1+\mathrm{j} 1.2$ (p.u.) and the rotor side is $0.5+\mathrm{j} 2$ (p.u.). The magnetizing reactance of the WG is 400 (p.u.). The impedance of the box-type transformer is $0.0625+\mathrm{j} 4.3333$ (p.u.).

The load flow analysis of the modified IEEE-30 test system is carried out when a wind farm is integrated. Table 3 illustrates the load flow results before and after applying aggregation method. Compared with that of distributed WGs in the wind farm, the voltage of the added Bus 31 still can be derived accurately by using equivalent method, which verifies the effectiveness of wind farm aggregation.

\section{TABLE 3}

Voltage of the wind farm with different equivalent methods adopted

\begin{tabular}{ccccc}
\hline \multirow{2}{*}{$v_{w}(\mathrm{~m} / \mathrm{s})$} & $P_{m, i, \Sigma}$ & 64 single WGs & 8 equivalent WGs & \begin{tabular}{c} 
Relative error of \\
two methods \\
\cline { 3 - 4 }$(\%)$
\end{tabular} \\
\hline 5 & 0.061321 & 0.997482 & 0.997483 & 0.00010 \\
8 & 0.315380 & 0.996669 & 0.996681 & 0.00120 \\
10 & 0.613770 & 0.993508 & 0.993539 & 0.00312 \\
12 & 0.883314 & 0.988544 & 0.988551 & 0.00071 \\
14 & 0.958163 & 0.986775 & 0.986778 & 0.00030 \\
\hline
\end{tabular}

By using the wind farm aggregation method, the WGs along one row would be replaced by an equivalent WG with a virtual line. In terms of these 8 equivalent WGs, PQ model, Simplified RX model and Three-Node model are carried out into the calculation of load flow for comparison. Table 4 shows the results of load flow calculation with PQ Model, RX Model and Three-Node 
Model adopt when the value of wind speed varies from $5 \mathrm{~m} / \mathrm{s}$ to $14 \mathrm{~m} / \mathrm{s}$.

\section{TABLE 4}

Voltage of the wind farm with different models adopted

\begin{tabular}{ccccc}
\hline \multirow{2}{*}{$\begin{array}{c}v_{w} \\
(\mathrm{~m} / \mathrm{s})\end{array}$} & $P_{m, i, \Sigma}$ & PQ Model $^{[5]}$ & Simplified RX Model $^{[5]}$ & Three-Node Model \\
\cline { 3 - 5 } 5 & 0.061321 & 0.997483 & 0.997483 & 0.997483 \\
8 & 0.315380 & 0.996681 & 0.996681 & 0.996681 \\
10 & 0.613770 & 0.993539 & 0.993539 & 0.993539 \\
12 & 0.883314 & 0.988551 & 0.988551 & 0.988551 \\
14 & 0.958163 & 0.986778 & 0.986778 & 0.986778 \\
\hline
\end{tabular}

Obviously, the results of three methods have no difference with each other. Together with the results of Table 3, it shows that the Three-Node Model of WG proposed in this paper for is quite feasible for steady-state analysis of power systems with the aggregated wind farm.

To compare the computation efficiency, the detailed iterative processes of PQ Model, RX Model and Multiple-Node Model are demonstrated as Figure 6, which is calculated at the value of $v_{w}$ being equal to $8 \mathrm{~m} / \mathrm{s}$. In addition, the calculation time of PQ Model and RX Model is $390 \mathrm{~ms}$ and $203 \mathrm{~ms}$ and the Multiple-Node Model is $141 \mathrm{~ms}$, which is carried out on a PC with Intel Core i5 2.9-GHz processor and 8GB of memory.

Since in the RX Model the iteration of the slip $s$ has to be executed together with voltage, the iterative process of load flow could be quite time-consuming. However with Three-Node Model, as wind farm is transferred into equivalent WGs on some general buses, the iteration process should be fast and have the square convergence property of Newton-Raphson algorithm. Figure 6 verifies this analysis, and reveals the high computation efficiency of Multiple-Node Models. 


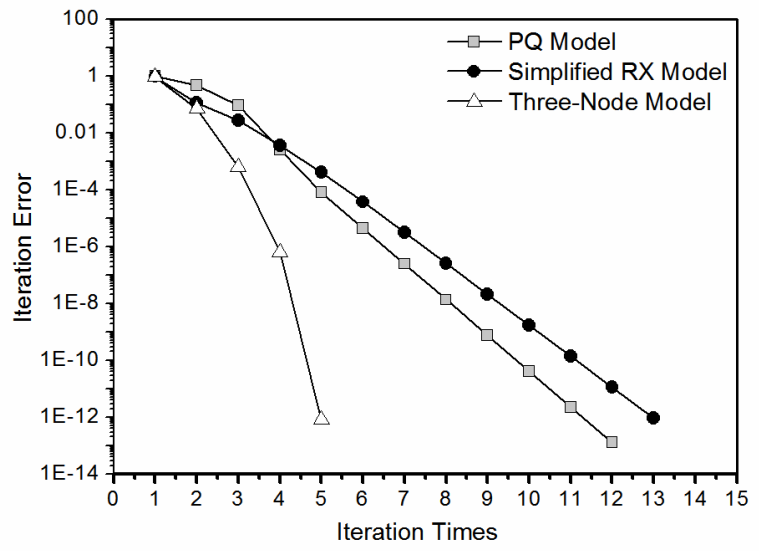

Figure 6. The iterative process of load flow calculation by using proposed models

\section{Conclusions}

In this article, a Three-Node Model is proposed for asynchronous WG. This model converts the induction generator circuit into several power system buses and branches to transmit wind power. From practical point of view, a wind farm aggregation method is introduced. The case study verified the feasibility and accuracy of the proposed model. In addition, the efficiency of this model is clearly demonstrated by comparing with existing models. The dominating advantages of this model can be concluded as follows:

In the first place, the Three-Node Model properly described a fixed speed induction generator. It has confirmed that the accuracies of this model and other existing models are equally matched.

What count is the convenience and flexibility to have this model performed in universal load flow programs. This model eliminates the iteration process of rotor slip or other redundant calculations and helps unburden the computation complexity.

In practice, the combination of Three-Node Model and wind farm aggregation method further improves the superiority of its application to the load flow analysis incorporating wind farms.

\section{Acknowledgment}

This work was supported by National Natural Science Foundation of China (51277141) and National High Technology Research and Development Program of China (863 Program, No. 2011AA05A103). 


\section{References}

[1] Xie, K.G., Li, Y.N., and Li, W.Y., "Modeling wind speed dependence in system reliability assessment using copulas," IET Renew. Power Gener., Vol. 6, No. 6, pp. 392-399, November 2012.

[2] Muyeen, S.M., Hasanien, M.H., and Al-Durra, A., "Transient stability enhancement of wind farms connected to a multi-machine power system by using an adaptive ANN-controlled SMES," Energy Convers. Manage., Vol. 78, No. 1, pp. 412-420, February 2014.

[3] Cadirci, I., and Ermis, M., "Double-output induction generator operating at sub synchronous and super synchronous speeds: steady-state performance optimization and wind-energy recovery," IEE Proceedings-Electric Power Applications., Vol. 139, No. 5, pp. 429-442, July 1992.

[4] Pecas Lopes, J.A., Barbosa, F.P.M., and Pidre, J.C., "Simulation of MV distribution networks with asynchronous local generation sources," IEEE Proc. Electro-Technical Conf., Vol. 2, pp. 1453-1456, May 1991.

[5] Feijóo, A., and Cidrás, J., "Modeling of wind farms in the load flow analysis," IEEE Trans. Power Syst., Vol. 15, No. 1, pp. 110-115, February 2000.

[6] Feijóo, A., and Cidrá, J., "Corrections to modeling of wind farms in the load flow analysis," IEEE Trans. Power Syst., Vol. 16, No. 4, pp. 955, November 2001.

[7] Divya, K. C., and Rao, P. S. N., "Models for wind generator generating systems and their application in load flow studies," Elec. Power. Syst. Res., Vol. 76, pp. 844-855, June 2006.

[8] Feijóo, A., "On PQ Models for asynchronous wind generators," IEEE Trans. Power Syst., Vol. 24, No. 4, pp. 1890-1891, November 2009.

[9] Wang, L., Lin, Y.H., and Chen, Y.T., "Load-flow analysis of a wind farm containing multiple wind-driven wound-rotor induction generators with dynamic slip control using RX Models," IEEE Trans. Sustainable Energy., Vol. 2, No. 3, pp. 256-264, July 2011.

[10] Fuerte-Esquivel, C.R., Tovar-Hernández, J. H., Gutierrez-Alcaraz, G., Cisneros-Torres, F., et al, "Discussion of 'Modeling of wind farms in the load flow analysis'," IEEE Trans. Power Syst., Vol. 16, No. 4, pp. 951-952, November 2001.

[11] Castro, L.M., Fuerte-Esquivel, C.R., and Tovar-Hernandez, J.H., "Solution of Power Flow with Automatic Load-Frequency Control Devices including Wind Farms," IEEE Trans. Power Syst., Vol. 27, No. 4, pp. 2186-2194, November 2012.

[12] Eminoglu, U., "A new model for wind turbine systems," Elect. Power Compon. Syst., Vol. 37, No. 10, pp.1180-1193, April 2009.

[13] Eminoglu, U., "Modeling and application of wind turbine generating system (WTGS) to distribution systems," Renewable Energy, Vol. 34, No. 11, pp. 2474-2483, November 2009.

[14] Ali, M., Ilie, I.S., Milanovic, J.V., and Chicco, G., "Wind farm model aggregation using probabilistic clustering”, IEEE Trans. Power Syst., Vol. 28, No. 1, pp. 309-316, February 2013.

[15] Muljadi, E., Butterfield, C.P., Ellis, A., Mechenbier, J., et al, "Equivalencing the collector system of a large wind power plant," IEEE Power Engineering Society General Meeting, Canada, pp. 1-9, June 2006.

[16] Power System Test Case Archive. [Online]. Available: http://www.ee.washington.edu/research/pstca/. 


\section{List of figures}

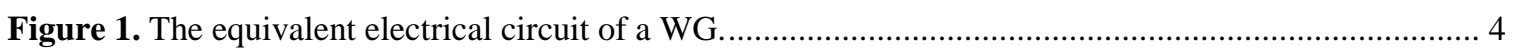

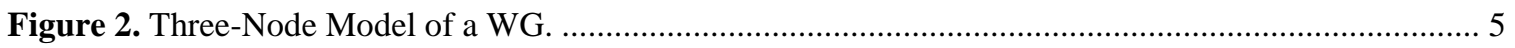

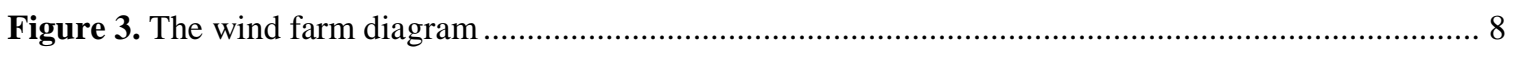

Figure 4. The equivalent model of the whole wind farm........................................................................10

Figure 5. The diagram of the modified IEEE-30 test system..................................................................10

Figure 6. The iterative process of load flow calculation by using proposed models ...................................13 